Recepción: 20 / 04 / 2017

Aceptación: 20 / 05 / 2017

Publicación: 15 / 07 / 2017
Ciencias de la educación

Artículo Científico

\title{
Musicoterapia como técnica de lenguaje oral en niños autistas
}

\section{Music therapy as an oral language technique in autistic children}

\section{A musicoterapia como uma técnica de linguagem oral em crianças autistas}

Nancy J. Rodríguez-Calderón ${ }^{\mathrm{I}}$ nancyrodriguezcalderon@ hotmail.com

Sonia B. Gómez-Vergara II soniabvergara@gmai.com Adriana M. Intriago Rosado III adrimintriago@gmail.com

Martha A. Ayala Paredes IV marthaayalaa@gmail.com

Correspondencia: nancyrodriguezcalderon@hotmail.com

\footnotetext{
${ }^{\mathrm{I}}$ Docente, Universidad de Guayaquil, Guayaquil, Ecuador.

II Docente, Universidad de Guayaquil, Guayaquil, Ecuador.

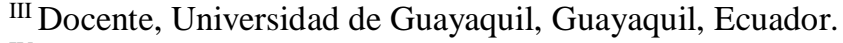

${ }^{I V}$ Docente, Universidad de Guayaquil, Guayaquil, Ecuador.
} 


\section{Resumen}

Este trabajo abarca el proceso de la musicoterapia y su función como técnica del lenguaje oral para desarrollar habilidades verbales; durante esta intervención se utilizó el método canto percusión que es un entrenamiento motor y auditivo acompañado del sonido de un par de tambores o maracas, mientras el terapeuta introduce palabras o frases en forma de canción. Tiene como objetivo describir el trabajo experimental demostrando que los autistas se sienten atraídos por los estímulos auditivos, recordando que la música es un medio de rehabilitación que ayuda a relajar, eso es lo que percibimos en los niños que asisten a la Fundación Comunicar, sitio donde realizamos nuestro estudio, mediante criterios de inclusión, se seleccionó pacientes que posean comprensión, que tengan afinidad con la música, y comunicación no verbal, los resultados se expresaron con la predilección de los niños hacia los estímulos musicales que redundaron en la manifestación comunicativa mediante el lenguaje oral en un $70 \%$ aproximadamente, mientras que el $30 \%$ restante, lo hicieron a través de intenciones comunicativas.

Palabras claves: Habilitación; rehabilitación; comunicación; conducta. 


\section{Summary}

This work covers the process of music therapy and its role as an oral language technique to develop verbal skills; During this intervention the percussion singing method was used which is a motor and auditory training accompanied by the sound of a pair of drums or maracas, while the therapist introduces words or phrases in song form. It aims to describe the experimental work demonstrating that autistic people are attracted to the auditory stimuli, remembering that music is a means of rehabilitation that helps to relax, that is what we perceive in the children who attend Fundación Communicacion, site where We carried out our study, using inclusion criteria, we selected patients who possess understanding, who have affinity with music, and nonverbal communication, the results were expressed with the children's preference for the musical stimuli that resulted in the communicative manifestation through Oral language in about $70 \%$, while the remaining $30 \%$ did so through communicative intentions.

Keywords: Habilitation; rehabilitation; communication; conducts. 


\section{Resumo}

Este trabalho aborda o processo de terapia de música e seu papel como uma técnica de linguagem oral para desenvolver habilidades verbais; cantando método de percussão é uma formação motora e auditiva acompanhada pelo som de um par de tambores e maracas, enquanto o terapeuta introduz palavras ou frases em canção foi usada durante este procedimento. Destina-se a descrever o trabalho experimental que demonstra que as pessoas autistas são atraídos a estímulos auditivos, lembrando que a música é um meio de reabilitação que ajuda a relaxar, que é o que vemos em crianças que frequentam Comunique Foundation, lugar realizamos nosso estudo, por critérios de inclusão, pacientes que possuem entendimento, que têm uma afinidade com música e comunicação não-verbal foi selecionado, os resultados foram expressos com a predileção das crianças aos estímulos musicais resultou em demonstração comunicativa por linguagem oral em cerca de $70 \%$, enquanto que os restantes $30 \%$ fez através intenções de comunicação.

Palavras-chave: Habilitação; reabilitação; comunicação; comportamento. 


\section{Introduccion.}

El autismo es uno de los males del comportamiento infantil que al no encontrar su origen se ha incrementado de manera alarmante sin que su avance se le pueda detener. En 1991 fue introducido el término autismo por el Suizo Bleuler quien lo describió como el rechazo al contacto con otras personas y un trastorno en su relación con la realidad.

Maseda, M. (2012) en su texto El autismo y las emociones manifiesta lo siguiente en cuanto al término autismo: "La palabra autismo fue utilizada por primera vez en 1911 por Eugene Bleuler, un psiquiatra suizo, que introdujo el término Autismo, del griego, "propio", "sí mismo" para designar uno de los rasgos propios de la esquizofrenia en adultos: la pérdida de contacto con la realidad y, como consecuencia, una gran dificultad para comunicarse con los otros”. (p. 14)

En términos generales se caracteriza por la intensa concentración de una persona en su mundo interior y la progresiva pérdida de contacto con la realidad exterior en su diario accionar, dificultad para el juego simbólico, evita mirar a los ojos, tiene escasas expresiones faciales y presenta descontrol de sus emociones y ansiedades; refiriéndose a la comunicación tiene un desarrollo lento en el lenguaje expresivo y receptivo. Las características principales del autismo son: diferencias en interacciones sociales recíprocas, comunicación y conducta. En la interacción social que se manifiesta generalmente con dificultad interpretando el lenguaje no verbal.

Existen diversos tipos de autismo entre los que se citan - el Síndrome de asperger, donde la persona afectada presenta ciertas dificultades tanto de interacción social como en la comunicación. Por otra parte, se observa un interés extremo en determinadas actividades o acciones, - Síndrome desintegrativo de la niñez, este tipo de autismo se caracteriza por un marcado retroceso en un conjunto de áreas de funcionamiento, adquiridas previamente por la persona. Como consecuencia, 

ésta pierde la capacidad de movimiento, la habilidad del lenguaje, la interacción social, el control de los esfínteres, --síndrome de Rett, este síndrome se caracteriza por un retraso profundo en la aprehensión del lenguaje y la coordinación motora, esta afección no emerge con el nacimiento del niño, sino que se presenta durante el segundo o tercer año de vida, -Trastorno del desarrollo no especificado, en esta categoría se incluyen aquellas afecciones severas vinculadas con la dificultad para desarrollar ciertas capacidades de comunicación o interacción social.

Varios estudios han demostrado que las personas con autismo presentan atracción a los estímulos sonoros cuando estos son musicales, por ello la musicoterapia se ha utilizado para mejorar las conductas sociales y comunicativas de personas con este trastorno.

Según Gigena, F (2013) en su texto Autismo y la Música sostiene:

“La música es vivida por el niño como algo que no es amenazador, por el contrario, es una situación que le brinda experiencias de seguridad" (p. 27)

El autor del texto nos indica que la música para el niño es una experiencia agradable, que no le asusta, más bien lo tranquiliza emocionalmente, por lo que el trabajo terapéutico se vuelve para el sujeto una aventura que deseará repetir más no una obligación.

El objetivo de la musicoterapia en este estudio fue dar inicio al lenguaje oral y desarrollar intenciones comunicativas, el mismo que se cumplió en la mayoría de los niños que recibieron este tipo de intervención. 


\section{Materiales y métodos (Metodología).}

Los materiales que se utilizaron fueron las fichas de evaluación, que respondieron a los objetivos planteados en la investigación y que fueron diseñadas para despejar todas las interrogantes sobre: "La musicoterapia como técnica del lenguaje oral en niños autistas; un instrumento de percusión que el niño prefiera, puede ser: maracas, tambores, platillos, castañuelas, etc. Además se utilizó laptop y videos musicales que ayudaron al aprendizaje del niño autista, y para la evaluación nos sirvieron:

- Historias clínicas, fichas de evaluación del lenguaje, encuestas a diversos profesionales de la salud, etc.

El diseño de la investigación fue de tipo descriptivo, en el que la técnica de habilitación para el lenguaje oral es analizada de acuerdo a los avances. Experimental en el que se demuestra que la aplicación de la técnica de canto percusión de la musicoterapia en niños culmina con la comunicación oral y por ende produciéndose la habilitación que es el motivo de la habilitación.

El método que se usó es el Canto - Percusión que consiste en la combinación oral, auditiva, motora y visual de los niños con autismo, más claramente se refiere a que el niño al iniciar su sesión terapéutica observa y escucha un video musical que contenga la información que se desea instaurar en él y que está basado en sus necesidades; luego se realiza una entonación del ritmo que escuchó anteriormente acompañada por el sonido del instrumento de percusión. En ese momento el terapeuta introduce palabras y frases muy cortas con pronunciación sencilla en forma de canción mientras toca el instrumento de percusión de manera acompasada. 


\section{Resultados.}

Para evaluar los resultados de la Técnica de Musicoterapia se aplicó el test de Zimmerman que mide la comprensión auditiva y la habilidad verbal de los niños desde 1 año 6 meses hasta los 8 años y que se evalúa de 0 a 4 puntos.

- En cuanto al rango de habilidad verbal los 25 niños autistas no verbales de seis años, en la evaluación inicial, es decir, antes de la aplicación de la técnica mostraron un puntaje de cero. Mientras que en la evaluación final estos mismo niños tuvieron como resultado dos puntos.

- Los 15 niños autistas no verbales de ocho años, en la evaluación inicial mostraron un puntaje de cero y en la evaluación final se mantuvo el mismo puntaje.

- $\quad$ En el rango de comprensión auditiva los 25 niños autistas no verbales de seis años en la evaluación inicial tuvieron un puntaje de tres y en la evaluación final estos niños se mantuvieron en el mismo puntaje.

- Con respecto a los 15 niños autistas no verbales de ocho años de edad, dentro de este rango, en la evaluación inicial mostraron un puntaje de 2 y en la evaluación final mostraron el mismo puntaje.

- Para obtener los resultados se aplicó el test de Zimmerman porque dicho test está dirigido a evaluar la habilidad verbal, que es el área que ha mejorado luego de la aplicación de la técnica.

- $\quad$ En cuanto a los resultados podemos decir que los pacientes que recibieron musicoterapia pasaron de comportamientos agresivos a comportamientos más cercanos y con un poco más de contacto físico con otras personas.

- $\quad$ Es importante resaltar que la Musicoterapia tiene mejores resultados cuanto más temprana es la edad del niño autista que la recibe. 
- En el área de atención, los niños mejoraron notablemente ya que durante cada terapia ponían mucha atención lo que se reflejaba en el aprendizaje de cada música que se les enseñaba.

- $\quad$ Otra de las situaciones que tiene una gran relevancia es que el tipo de música que se va a utilizar esté cargada de conocimiento, del cual el niño autista pueda aprender algo, como el esquema corporal, a reconocer frutas, animales, entre otras, Es decir, que pueda cubrir deficiencias que el niño autista presente y sobre todo realizar expresión corporal durante la musicoterapia porque es lo que el niño autista va a imitar durante la sesión.

- $\quad$ Para que este método de resultados adecuados se debe introducir palabras sencillas durante la musicoterapia, para que el niño tenga mayor facilidad en sus intentos comunicativos verbales, así como la duración de una sesión de musicoterapia debe depender exclusivamente de la capacidad de atención y colaboración del paciente.

\section{Discusión.}

Actualmente existe un número considerable de niños con autismo, como se mencionó anteriormente al tomar datos referenciales de la Organización Mundial de la Salud; ciertamente uno de los grandes deseos de las familias es que el niño tenga algo de lenguaje oral, aunque la comunicación no siempre está ligada al lenguaje oral, la obtención del mismo representa la adquisición de otras habilidades del niño como la comprensión de la información entregada al interlocutor; y la expresión.

Se pretende que la habilitación se base en las necesidades específicas de este grupo de pacientes para que mejoren la comunicación adquiriendo el lenguaje oral a través de la técnica de musicoterapia con el método canto percusión y aunque no es una articulación oral totalmente 
adecuada, es el inicio del lenguaje oral que con terapias posteriores para la articulación verbal puede mejorar su inteligibilidad

Este estudio nos ofrece un nuevo camino para mejorar la intervención dedicada a la adquisición de habilidades verbales en niños con autismo, porque es una terapia de muy poco costo en cuanto al uso de materiales tanto para el terapeuta que la imparte como para los padres del niño que la recibe.

\section{Bibliografía.}

Gigena, F. (2013). Autismoy La Música. España: Universitas

Martínez Martin, M., Cuesta Gómez, J. (2013). Trastornos Del Espectro Autismo. España: Alfaomega Altaria.

Maseda, M. (2012). El Autismo Y Las Emociones. España: Universitat De Barcelona

Vaillancourt, G. (2010). Música Y Musicoterapia. España: Narcea. 